\title{
Características de la violencia de género en la Universidad de Valencia
}

\section{Characteristics of gender violence at the University of Valencia}

\author{
Francisco González Sala ${ }^{1}$ y Belén Mora Valero ${ }^{2}$ \\ ${ }^{1}$ Universidad de Valencia, España \\ ${ }^{2}$ Programa Prevención Apoyo a Menores y Familias. Asociación de Voluntarios de Acogimiento Familiar. Valencia, España.
}

Disponible online 31 de agosto de 2014

\begin{abstract}
El presente estudio tiene como objetivo conocer la prevalencia de la violencia de género en la universidad de Valencia, el perfil de la víctima, recursos y creencias sobre la violencia en la comunidad universitaria. La muestra está formada por 3.404 sujetos pertenecientes al personal docente e investigador, el personal de administración y servicios y estudiantes. Los resultados apuntan una incidencia de la violencia de género del 20.03\%. El perfil de la víctima es el de mujer, estudiante, sin hijos, con una percepción del riesgo leve o moderado, que no denuncia y con repercusiones a nivel psicológico. El 86.99\% considera necesario que la universidad disponga de un servicio de atención a la violencia de género, y que se habiliten protocolos de actuación y programas de prevención. Entre el $25 \%$ y el $40 \%$ no considera violencia de género conductas relacionadas con el control y el maltrato psicológico. Resulta necesario implementar programas de prevención y atención de la violencia de género.
\end{abstract}

Palabras clave: Violencia de género; Universidad; Creencias; Recursos.

The present study attempted to determine the prevalence of gender-based violence at the University of Valencia, the victim profile, resources, and beliefs about violence in the university community. The sample consisted of 3404 participants from the research and teaching staff, the administration and services personnel, and students. The results suggest an incidence of gender violence of $20.03 \%$. The victim profile is a women student who is childless, at a perceived slight or moderate risk, who does not go to the police, but who experiences psychological repercussions from gender violence. In total, $86.99 \%$ of participants believe that the university has a gender violence service available and that action protocols and prevention programs have been implemented. Between $25 \%$ and $40 \%$ do not consider controlling behaviour and psychological abuse to constitute domestic violence. Gender violence prevention programs should be implemented.

Key words: Gender violence; University; Beliefs; Resources.

Correspondencia: Francisco González Sala. Dpto. Psicología Evolutiva y de la Educación. Universidad de Valencia. Avda. Blasco Ibáñez, 21. 46010 -Valencia, España. E-mail: Francisco.Gonzalez-Sala@uv.es. E-mail de la co-autora: bemova@hotmail.com 
La violencia de género afecta a mujeres de todas las edades, culturas, clases sociales y niveles educativos, manifestándose en diferentes contextos sociales, familiares y laborales y en todas las partes del mundo (Menéndez, Pérez y Lorente, 2013; Nabors, Dietz y Jasinski, 2006; Oliver y Valls, 2004; Sanmartín, Iborra, García y Martínez, 2010). Los datos sobre mujeres asesinadas o denuncias por malos tratos son sólo la punta del iceberg de una realidad que dadas sus consecuencias, son una lacra social sobre la que se aplican multitud de recursos en su prevención e intervención. Según los resultados de la IV Macroencuesta de Violencia de Género (Observatorio Estatal de Violencia sobre la Mujer, 2011) un 10.9\% de las mujeres mayores de 18 años en España, manifestaba haber sido víctima de violencia de género en algún momento de su vida.

Investigar sobre la violencia de género en el ámbito universitario supone romper el silencio alrededor de las situaciones que se producen en este contexto. En este sentido, diferentes estudios han abordado la violencia de género en el contexto universitario (Bolanos, 2003; Eyre, 2000; Gross, Winslett, Roberts y Gohm, 2006; Oliver y Valls, 2004; Rentschle, 2000; Straus, 2004; Valls, Oliver, Sánchez, Ruíz y Melgar, 2007), bien para determinar su incidencia e impacto sobre los estudiantes, bien para valorar diferentes programas de prevención y atención a las víctima que se llevan a término, como serían los casos de las universidades de Cambridge y Oxford en el Reino Unido o las de Harvard, Yale y Standford en Estados Unidos. En España el trabajo de Valls, Flecha y Melgar (2008) en cuatro universidades catalanas, destaca la importancia de crear servicios específicos de atención a la víctima dentro de la propia universidad, si bien, este tipo de medidas son prácticamente inexistentes en las universidades españolas.

Muñoz-Rivas, Graña, O’Leary y González (2006) encuentran que uno de cada cinco universitarios ha experimentado abusos de tipo físico en la relación de noviazgo, y más de la mitad ha sufrido violencia psicológica, principalmente agresiones de tipo verbal y acciones dirigidas al control de la pareja. Ferrer, Bosch, Ramis y Navarro (2006) apuntan que las variables sociodemográficas, familiares y formativas explican entre el $5.5 \%$ y el $13.4 \%$ de las creencias y actitudes hacia las mujeres y el uso de la violencia en población universitaria. Según Straus (2004) un 29\% de universitarios pertenecientes a varios países habían cometido algún tipo de agresión sobre su pareja. Smith, White y Holland (2003) un $77.5 \%$ de estudiantes universitarias en Estados Unidos en último año de carrera, había sufrido algún tipo de violencia física o sexual por sus parejas.

En el contexto español Valls (2008) encuentra que un 13\% de los estudiantes universitarios conoce algún caso de violencia de género, porcentaje que se incrementa hasta el $65 \%$ cuando se analizan las respuestas dadas a conductas definidas como violencia de género. Entre un 20\% y un 33\% de los universitarios no consideran violencia de género conductas relacionadas con el dominio o control de la mujer, prohibir hablar con otras personas, hacer observaciones desagradables sobre el aspecto físico de la pareja, perseguir a la pareja, exigirle dar explicaciones de con quién y dónde va. En un 66\% la respuesta de la víctima fue no denunciarlo, una cuarta parte lo mantuvo en secreto y el resto optó por contárselo a alguien. El 92\% desconoce si su universidad dispone de algún servicio específico donde acudir como víctima. Un $85 \%$ piensa que es necesario habilitar este tipo de recursos y para un $86 \%$ resulta necesario abordar esta temática en actividades formativas como seminarios y cursos. Según Vázquez, Torres, Otero, Blanco y López (2010) un 6,3\% de las mujeres universitarias españolas habría sufrido maltrato psicológico en su relación de pareja.

Los recursos que dispone la Universidad de Valencia relacionados con la violencia de género son una serie de conferencias, jornadas, actos y manifestaciones de condena y rechazo hacia este tipo de violencia y concursos de relatos que se organizan principalmente entorno al día internacional contra la violencia a las mujeres, todo ello a través de la unidad de igualdad (Unidad de Igualdad, 2012). El único programa que atiende directamente a alguno de los sujetos directos o indirectos de la violencia de género es el programa de investigación, formación e intervención con hombres penados por violencia contra la mujer, dentro del programa Contexto, desarrollado por Lila et al. (2010) desde el año 2006.

Entre las causas de la violencia de género la literatura científica ha identificado factores ideológicos, desigualdades de poder en la relación o el sexismo benévolo en las mujeres (Herrera, Expósito, Moya y Houston, 2012), aspectos educacionales y sociales (Hirigoyen, 2006), creencias, mitos y estereotipos sobre la violencia y actitudes sexistas en la relación (Bosch-Fiol y Ferrer-Pérez, 2012; Montilla, Pérez-Cordón y Montes-Berges, 2010; Peters, 2008), además de diferentes factores que contribuyen a perpetuarla, como la percepción y los prejuicios de la sociedad hacia las mujeres maltratadas (Expósito y Herrera, 2009; Montilla, Pérez, Castillo-Mayén y Montes-Berges, 2012). Según la Comisión Europea (2001) gran parte de los europeos consideran la predisposición genética como causa de la violencia de género, un $96 \%$ la atribuyen al consumo de alcohol y un $25 \%$ consideran que la mujer ha de ocuparse del hogar y el hombre de ganar el sustento. Según Heise, Ellsberg y Gottemoeller (1999) los factores de riesgo asociados a la violencia de género desde un modelo ecológico son de índole social - roles de género rígidos o normas que dan por hecho el control del hombre sobre la mujer -, factores comunitarios como sería el aislamiento social y familiar de las mujeres o la pobreza, factores relacionales como serían el control del dinero y la toma de decisiones por el hombre dentro de la familia, y factores individuales como serían el uso del alcohol, interiorización de valores y roles tradicionales asociados al género o ser testigo de violencia en la familia de origen.

El objetivo del presente estudio es determinar la incidencia de la violencia de género en la Universidad de Valencia, 
teniendo en cuenta sus tres actores principales, el personal docente e investigador, el personal de administración y servicios y los estudiantes. En este sentido nos interesa conocer la incidencia y las características de la violencia de género, los recursos, la percepción de los mismos y necesidades, así como las creencias sobre la violencia de género, con el fin último de habilitar recursos, dentro de la universidad, para abordar el estudio, prevención y atención de las víctimas de la violencia de género que pertenezcan al ámbito universitario.

\section{Método \\ Participantes \\ La muestra está formada por un total de 3.404 sujetos, de los cuales 252 pertenecen al Personal de Administración y Ser- vicios (PAS) lo que supone un $13.59 \%$ del total de este colec- tivo en la Universidad de Valencia, 266 al Personal Docente Investigador (PDI) lo que supone el $6.91 \%$ del total de PDI en la Universidad y 2.886 estudiantes, un $4.92 \%$ de los estu- diantes matriculados en el curso 2012-2013. De la muestra de estudiantes 557 son de $3^{\circ}$ ciclo, 1.037 de $2^{\circ}$ ciclo y 1.292 de $1^{\mathrm{o}}$ ciclo. Con respecto al género $703(20.65 \%)$ son hombres y $2.554(79.35 \%)$ son mujeres. En cuanto a la edad, 2.018 sujetos tienen entre 18 y 25 años, 657 entre 26 y 35 años y 729 más de 36 años. Con respecto al nivel socioeconómico 700 sujetos son de un nivel bajo o muy bajo, 2.696 de nivel medio y 208 alto o muy alto.}

\section{Instrumento}

Se ha empleado la "Encuesta de Violencia de Género en el Ámbito Universitario", elaborada para el presente estudio y revisada por cinco expertos en violencia de género, alcanzando un consenso entre jueces según el valor de la prueba Kappa de .79 y .85 . Ésta consta de un total de 32 ítems, algunos de los cuales son de respuesta única y otros de respuesta múltiple. La encuesta está dividida en cuatro apartados: (a) Datos sociodemográficos: recoge el estamento y el campus universitario de pertenencia, sexo, edad, nivel cultural y socioeconómico; (b) situación de la violencia de género en el ámbito universitario: incluye la incidencia de la violencia de género en su entorno más próximo, información referente a si se conoce algún caso de violencia de género dentro de la universidad y las características del mismo; (c) recursos de la universidad: se valoran los recursos existentes, si son o no suficientes, necesarios o hay que habilitar otros y cómo reaccionarían los encuestados ante una situación de violencia dentro de la universidad y (d) consideraciones respecto a la violencia de género: se valora la importancia de la violencia de género en la sociedad, las consideraciones sobre qué es y las causas de la misma y qué tolerarían o perdonarían por amor.

\section{Procedimiento}

Tras elaborar el instrumento para la recogida de información se pasó a solicitar los permisos para enviarlo a todo el colectivo (PDI, PAS y Estudiantes) de la Universidad de Valencia. Para garantizar el anonimato del encuestado, una vez cumplimentada la encuesta, se generaba un número único interno por encuesta y persona, borrando el programa los datos de usuario del encuestado, todo esto se gestionó a través del equipo de informáticos de la Facultad de Psicología y de la Universidad de Valencia. Por último, se pasó al envío de las encuestas a toda la comunidad universitaria, al análisis de resultados y a su posterior interpretación. La encuesta fue contestada de forma voluntaria, siendo el tiempo aproximado para cumplimentarla entre 10 y 15 minutos.

\section{Análisis estadístico}

El análisis de resultados, en este caso recuento de frecuencias parciales y totales, se realizó a través del paquete estadístico SPSS 19.

\section{Resultados \\ Situaciones de violencia de género en el ámbito universi- tario}

Mil doscientas veintisiete personas $(36.05 \%)$ vinculadas con la universidad de Valencia responden que han sufrido o han sido testigos en su entorno más próximo de una situación de violencia de género. Al preguntarles si conocen algún caso de violencia de género dentro de la universidad un 20.03\% (682 personas) responden que sí. Estos se caracterizan porque en 638 casos la víctima era una mujer y en 44 casos un hombre. De estos, 47 pertenecían al PDI, 73 al PAS, 386 eran estudiantes y en 176 era un miembro externo a la universidad. Con respecto al agresor, en 43 casos era una mujer y en 639 un hombre, de los que 31 pertenecían al PAS, 60 al PDI, 81 eran estudiantes, 455 personas externas a la universidad y en 55 casos se desconoce si el agresor tenía vinculación con la universidad.

En un $19.80 \%$ la víctima tenía hijos. En un $36.65 \%$ la persona no consideró que era víctima de una situación de violencia de género, siendo la percepción del riesgo por parte de la víctima leve en un $29.47 \%$, moderado en un $31.96 \%$, grave en un $21.84 \%$, muy grave en un $6.74 \%$ y en un $9.97 \%$ la víctima no percibió riesgo alguno.

Por lo que respecta a las medidas que tomó la víctima, lo más frecuente fue contárselo a alguien un $53.07 \%$ y romper la relación un $44.77 \%$. Otras actuaciones fueron denunciarlo un $20.67 \%$, acudir a un profesional o recurso de ayuda específico un $18.47 \%$, comunicarlo a la universidad un $6.89 \%$, cambiar de puesto de trabajo o pedir un traslado un $5.13 \%$. Destacar que en un $30.93 \%$ la víctima no tomó ningún tipo de medida y lo dejó pasar y que un $30.79 \%$ continuó con la relación de pareja.

Las razones por las que la víctima no denunció fueron que no le pareció tan grave y quería olvidar lo sucedido en un $30.79 \%$ y $30.35 \%$ respectivamente, dependencia emocional o económica en un $23.90 \%$, miedo a las represalias, sentimientos de culpabilidad y vergüenza entorno al 19\%, el resto de argumentos como estar en estado de shock a nivel emocional, 
desconfianza hacia el sistema de protección y/o judicial, motivos ideológicos, por los hijos y no saber a quién dirigirse están presentes entre un 12 y un $5 \%$.

Las principales consecuencias sobre la víctima fueron problemas de salud psicológica en un $62.02 \%$, repercusiones a nivel laboral en un $37.09 \%$, aislamiento social y problemas de salud física entre un $29 \%$ y un $22 \%$, y repercusiones económicas y cambios de lugar de residencia alrededor del $12 \%$.

Ante la pregunta acerca de si se tenía conocimiento de si alguna persona de la universidad había sufrido alguna situación de violencia dentro de la universidad o entre personas universitarias, la mitad de la muestra lo desconoce y un $36.19 \%$ señala conocer casos de desvalorizaciones, menosprecios en público o en privado por ser mujer, el resto de porcentajes se pueden consultar en la Tabla 1.

\section{Tabla 1}

Situaciones de violencia dentro de la universidad o entre personas universitarias

\begin{tabular}{lcc}
\hline Situación & Frecuencia & Porcentaje \\
\hline $\begin{array}{l}\text { Daños o amenazas a otros familiares o amigos } \\
\text { de la víctima }\end{array}$ & 71 & 2.09 \\
$\begin{array}{l}\text { Control de los ingresos económicos del hogar, } \\
\text { chantaje económico }\end{array}$ & 80 & 2.35 \\
$\begin{array}{l}\text { Daños apreciados por él/ella } \\
\text { Besos y caricias sin consentimiento }\end{array}$ & 124 & 3.64 \\
$\begin{array}{l}\text { Agresiones físicas } \\
\text { Prohibir o coaccionar para tener relaciones de }\end{array}$ & 140 & 4.11 \\
amistad & 211 & 6.20 \\
$\begin{array}{l}\text { Que le exijan obediencia o no pueda tomar } \\
\text { decisiones }\end{array}$ & 255 & 6.79 \\
$\begin{array}{l}\text { Haber sido vigilado-a, perseguido-a } \\
\text { Amenazas, intimidaciones... }\end{array}$ & 274 & 7.49 \\
$\begin{array}{l}\text { Chantaje emocional o coacciones para mantener } \\
\text { relaciones afectivas o sexuales }\end{array}$ & 314 & 8.05 \\
$\begin{array}{l}\text { Sentir incomodidad o miedo por comentarios, } \\
\text { llamadas, mensajes de texto intimidatorios... }\end{array}$ & 469 & 10.40 \\
$\begin{array}{l}\text { Control sobre la forma de vestir, peinarse, } \\
\text { horarios... }\end{array}$ & 473 & 13.78 \\
$\begin{array}{l}\text { Propagar rumores respecto a la vida sexual } \\
\begin{array}{l}\text { Invadir la intimidad de la persona leyendo sus } \\
\text { mensajes, correo electrónico, diario, agenda... }\end{array}\end{array}$ & 478 & 14.04 \\
$\begin{array}{l}\text { Desvalorizaciones, menosprecios en público o } \\
\text { en privado }\end{array}$ & 1.232 & 14.66 \\
Desconoce de alguna de estas situaciones & 1.726 & 50.71 \\
\hline
\end{tabular}

\section{Recursos de la universidad con relación a la violencia de género}

Entre las acciones que llevarían a término si sufriesen alguna situación relacionada con la violencia de género dentro de la universidad como empujones, besos y caricias sin consentimiento, desvalorizaciones, chantajes emocionales o coacciones para mantener relaciones afectivas o sexuales y sentirse vigilado, perseguido, que le exijan obediencia o amenazas, entre un $38.92 \%$ y $52.03 \%$ denunciaría dependiendo de la situación que se diese. Ante la prohibición de tener relaciones de amistad o que invadiesen su intimidad, la respuesta predominante sería dejar la relación en un $43.57 \%$. Todos estos datos vienen recogidos en la Tabla 2 .

A la pregunta de si conocen algún servicio o programa específico llevado a cabo por la universidad relacionado con la prevención de la violencia de género un $17.48 \%$ responde que sí, un $4.44 \%$ dice conocer un programa de intervención con maltratadores y un $4.55 \%$ en relación con la atención a las víctimas. El 78.70\% desconocen si existe alguno de estos servicios en la universidad de Valencia.

En relación con la puesta en marcha de servicios de atención e intervención con los sujetos de la violencia de género que tengan vinculación con la universidad, un $86.99 \%$ consideran necesario un servicio de atención a las víctimas, para un $44.89 \%$ sería necesario un servicio de atención a los hijos de las víctimas y un $40.16 \%$ con respecto a los agresores. Tan sólo, un $9.87 \%$ no consideran necesario la puesta en marcha de alguno de estos programas por parte de la universidad.

El $37.81 \%$ considera que la violencia de género no se aborda de forma suficiente por parte de la universidad frente al $16.48 \%$ que piensa que sí y un $45.71 \%$ lo desconoce.

Entre las acciones que la universidad podría habilitar de cara a tratar la violencia de género, un $79.23 \%$ apunta la necesidad de implementar protocolos de actuación ante la denuncia de una situación de violencia de género, un $76 \%$ consideran necesarios programas de prevención, formación e intervención, un $64.95 \%$ regular sanciones contra los-as agresores-as si pertenecen al ámbito universitario, alrededor del 39\% generar grupos de trabajo y realizar dípticos informativos, el $26.38 \%$ la

Tabla 2

Medidas que tomaría la persona ante situaciones de violencia dentro de la universidad. Frecuencias y porcentajes.

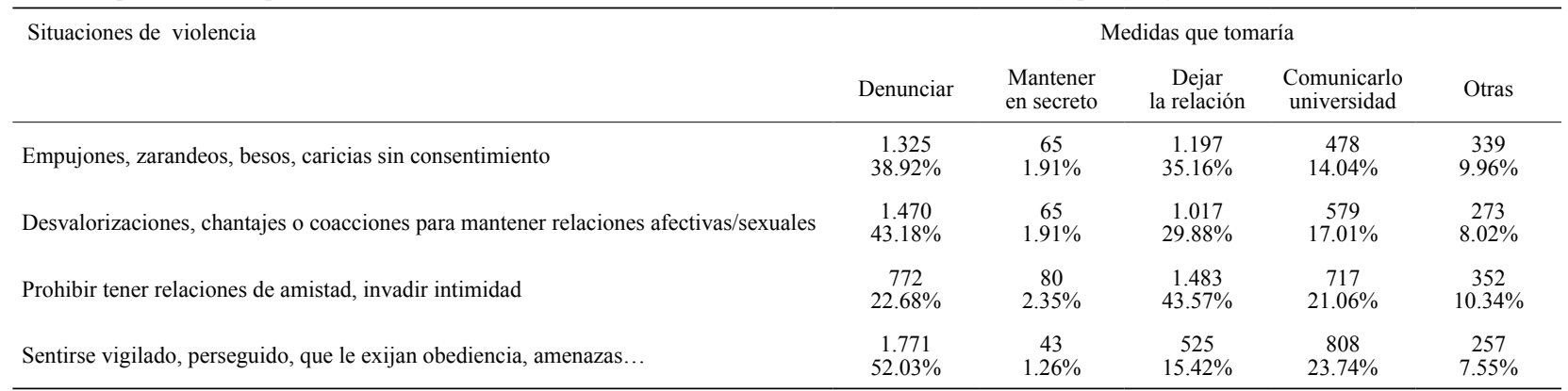


puesta en marcha de revistas o publicaciones especializadas, un $21.62 \%$ congresos científicos y un $13.31 \%$ apunta hacia otras medidas.

\section{Consideraciones respecto a la violencia de género por parte de la comunidad universitaria}

La importancia que tiene la violencia de género en la sociedad según la muestra, es muy importante en el $72.59 \%$, siendo importante en el $25 \%$. Menos de un 3\% considera que es poco o nada importante.

Ante la cuestión "Qué conductas consideran violencia de género dentro de una relación de pareja”, los porcentajes varían entre el $99 \%$ y el $60 \%$, siendo mayores en el caso de las conductas relacionadas con el maltrato físico y menores con respecto al maltrato psicológico. En la Tabla 3 vienen recogidos los datos para esta cuestión.

En relación con las causas de la violencia de género, principalmente la comunidad universitaria atribuye ésta a creencias machistas, estereotipos y roles de género en un $96.15 \%$ y factores socioculturales en un $86.40 \%$. Entre un $70 \%$ y un $67 \%$ también apuntan como causa la celopatía, el consumo de alcohol y drogas, la historia de vida del agresor y los problemas mentales. Con porcentajes inferiores al $29 \%$ se encuentran razones de tipo económico, desempleo, incompatibilidad de caracteres, predisposición genética o que la mujer haya abandonado el rol familiar tradicional.
A la pregunta de si tolerarían o perdonarían por amor en una sola ocasión, alguna situación que caracteriza a la violencia de género, sólo un $41.16 \%$ no toleraría ninguna de las situaciones que se describen en la Tabla 4.

Tabla 4

Situaciones que tolerarías o perdonarías por amor en una sola ocasión.

\begin{tabular}{lcc}
\hline Situaciones & Frecuencia & Porcentaje \\
\hline Que le dé un bofetón & 254 & 7.46 \\
Que le empuje & 335 & 9.84 \\
Que le insulte o desvalorice & 338 & 9.93 \\
Que controle su forma de vestir y de comportarse & 350 & 10.28 \\
Que le siga para ver con quién va o invada su & 408 & 11.99 \\
intimidad leyendo sus mensajes, correos, agenda... & & 12.75 \\
Que le prohíba hablar con alguien & 434 & 12.87 \\
Que le culpabilice de las cosas que le suceden & 438 & 13.13 \\
Que no le deje salir con sus amigos-as & 447 & 35.05 \\
Que le fuese infiel & 1.193 & 41.13 \\
Que sea celoso & 1.400 & 41.16 \\
No toleraría ninguna de estas situaciones & 1.401 & \\
\hline
\end{tabular}

\section{Discusión}

El perfil de persona que contesta a la encuesta es el de mujer, estudiante entre 18 y 25 años y de nivel socioeconómico medio. Mayoritariamente ha sido realizada por mujeres, un $79.35 \%$, frente al $20.65 \%$ de hombres que han contestado. Llama la atención que la proporción de hombres y mujeres en la Universidad de Valencia no es tan acusada, en concreto el

Tabla 3

Acciones consideradas violencia de género dentro de una relación de pareja.

\begin{tabular}{|c|c|c|}
\hline Acciones & Frecuencia & Porcentaje \\
\hline Hacer observaciones desagradables sobre la apariencia física & 2.070 & 60.81 \\
\hline Contar rumores respecto a la vida sexual o aspectos íntimos & 2.076 & 60.99 \\
\hline Controlar los ingresos económicos del hogar o de la familia & 2.085 & 61.25 \\
\hline Invadir la intimidad leyendo sus mensajes, agenda, registros... & 2.413 & 70.89 \\
\hline Romper cosas de la pareja & 2.431 & 71.42 \\
\hline Que la pareja exija saber con quién y dónde va & 2.443 & 71.77 \\
\hline Chantajear con contar secretos de la pareja o ex pareja & 2.683 & 78.82 \\
\hline Controlar la forma de vestir, peinarse... de la pareja & 2.751 & 80.82 \\
\hline Prohibir o coaccionar para tener relaciones de amistad & 2.837 & 83.30 \\
\hline Empujar, pellizcar & 2.885 & 84.75 \\
\hline Culpabilizar a la pareja de sus reacciones y desgracias & 2.920 & 85.78 \\
\hline Sentir incomodidad o miedo por comentarios, llamadas, mensajes insistiendo en mantener relaciones & 2.934 & 86.19 \\
\hline Castigar o maltratar a los hijos cuando está enfadado contigo & 3.012 & 88.48 \\
\hline Hacer sentir inferior a la pareja o ex pareja, tratarla como tonta & 3.105 & 91.22 \\
\hline Vigilar o perseguir a la pareja o ex pareja & 3.108 & 91.30 \\
\hline Exigir obediencia & 3.111 & 91.39 \\
\hline Perseguir insistentemente a la pareja o ex pareja & 3.116 & 91.54 \\
\hline Amenazar con matarse o matar a los hijos si lo abandona & 3.239 & 95.15 \\
\hline Humillaciones y desvalorizaciones como madre, mujer... & 3.265 & 95.92 \\
\hline Amenazar, intimidar & 3.288 & 96.59 \\
\hline Intentar mantener relaciones sexuales contra su voluntad & 3.303 & 97.03 \\
\hline Presiones y utilización de la fuerza para mantener relaciones afectivas o sexuales & 3.343 & 98.21 \\
\hline Golpear a la pareja o ex pareja & 3.368 & 98.94 \\
\hline
\end{tabular}


$41 \%$ de las personas vinculadas a la universidad son hombres, frente al 59\% que son mujeres, por lo que podemos concluir que el tema de la violencia de género ha despertado una mayor respuesta entre las mujeres que entre los hombres, lo cual puede ser debido a una mayor concienciación del problema entre ellas que entre ellos.

Por otro lado, destacar la presencia de situaciones de violencia de género en el entorno próximo de la persona que responde al cuestionario en un porcentaje a tener muy en cuenta, alrededor del $36 \%$, lo cual sólo hace que confirmar la incidencia de este tipo de violencia en nuestra sociedad.

Podemos concluir que la violencia de género es una realidad que afecta al contexto universitario, bien como víctimas de la misma, bien como agresores. Alrededor del 20\% de las personas encuestadas conocen o son testigos de un caso de violencia de género dentro la universidad. Porcentaje que puede ser mayor si tenemos en cuenta que un $36.19 \%$ reconoce haber sido testigo de desvalorizaciones, menosprecios en público o en privado, prevalencia que se encuentra por debajo de la que apunta Valls (2008) entorno al 65\%, o por Muñoz-Rivas et al. (2006) y Smith et al. (2003), siendo más parecido al que apuntan otros estudios como el de Straus (2004). Estos porcentajes no distan mucho de los encontrados en población procedente de servicios sociales, situándose alrededor del 37\% (González y Gimeno, 2009), lo que nos lleva a romper el estereotipo de que la violencia de género está presente en mayor medida en mujeres de bajo nivel cultural y económico (Burgués, Oliver, Redondo y Serrano, 2006).

El perfil de la víctima es el de mujer, estudiante universitaria, con baja percepción de ser víctima y del riesgo de la situación que padece, tomando como medidas ante la violencia contárselo a alguien o dejar la relación, con repercusiones negativas a nivel de salud mental y que no denuncia. Circunstancia esta última que está en consonancia con los datos del Instituto de la Mujer (2004), donde queda constancia que alrededor del $80 \%$ de las mujeres no presenta denuncia por malos tratos, y que en muchas ocasiones, una vez interpuesta se retira debido a que en muchas ocasiones la mujer quiere que termine el maltrato pero no la relación de pareja (Labrador, Rincón, de Luís y Fernández-Velasco, 2004; Medina, 2002).

Por lo que respecta al agresor, mayoritariamente son hombres, sin vinculación con la universidad, destacando que un $25.2 \%$ sí que tienen relación con esta institución, bien como estudiantes, PDI o como PAS, porcentaje muy a tener en cuenta. Las conductas relacionadas con la violencia psicológica son las más frecuentes dentro de la universidad, principalmente las desvalorizaciones o menosprecios en público o privado.

La denuncia es la respuesta más frecuente cuando se habla de situaciones relacionadas con la violencia de género dentro de la universidad. Aunque ésta opción sólo es considerada entre el $52 \%$ y el $38 \%$, por lo que podemos decir que más de la mitad no denunciaría estas situaciones, datos parecidos a los apuntados por Valls (2008). La mayoría de los entrevistados desco- noce si la universidad dispone de algún servicio de atención a la víctima de la violencia, a los hijos testigos de la misma, o a los maltratadores. La mayoría de los encuestados considera necesario la puesta en marcha de recursos de atención a las víctimas que tengan relación con la universidad, aspecto también aportado por Valls, Flecha y Melgar (2008), así como regular sanciones contra los agresores si pertenecen al ámbito universitario, o habilitar protocolos de actuación de cara a denunciar una situación de esta índole. Recursos todos ellos de los que no dispone actualmente la Universidad de Valencia. Otras actuaciones que se contemplan sería desarrollar programas de prevención y formación ante la violencia de género, actuaciones éstas que sí se realizan a través de la Unidad de Igualdad (2012) o a través de cursos de libre elección, así como la intervención con maltratadores, llevada a cabo mediante el programa Contexto que interviene con hombres penados por esta causa (Lila et al., 2010).

Existen dos aspectos que despiertan nuestra preocupación con respecto a las consideraciones sobre la violencia de género y a las repercusiones que esto puede tener en sus relaciones de pareja, en primer lugar acerca de que conductas son consideradas una forma de maltrato o no, y en segundo lugar qué se toleraría por amor.

Con respecto al primer punto, decir que entre un $40 \%$ y un $30 \%$ no considera violencia de género conductas como hacer observaciones desagradables sobre la apariencia física, contar rumores respecto a la vida sexual o aspectos íntimos de la pareja o expareja, controlar los ingresos económicos del hogar o de la familia, invadir la intimidad leyendo mensajes, correos privados o registrar las cosas personales de la pareja, romper cosas de la pareja o que le exijan saber con quién y donde va, y entre un $25 \%$ y $20 \%$ conductas como comparar a la pareja con otras mujeres para desvalorizarla, chantajear con contar secretos suyos y controlar la forma de vestir o peinarse, circunstancia que ya ha sido observada por Valls (2008), y que revela las dificultades a la hora de identificar un maltrato que no sea el físico (Meras, 2003; Valls, 2008).

En segundo lugar, la tolerancia hacia determinadas conductas y formas de pensar que guardan relación con la violencia de género, como son los celos, la infidelidad o el prohibir ir con amigos, las cuales serían toleradas por amor.

Todo ello nos lleva a plantearnos, en busca de la excelencia de la Universidad de Valencia contra la violencia de género, la necesidad de implementar dentro del currículum o por medio de actuaciones externas, programas de información y prevención de la violencia de género dirigidos a la comunidad universitaria, con vistas a detectar indicadores o situaciones de violencia de género y fomentar relaciones de pareja saludables basadas en el respeto, así como generar mecanismos que faciliten la comunicación y denuncia de situaciones de violencia. Por último, resulta necesario habilitar un servicio de atención a las víctimas de la violencia de género pertenecientes a la comunidad universitaria, bien coordinado desde la propia universidad 
o bien por un servicio externo, como pudiera ser el Instituto de la Mujer de Valencia perteneciente a la Dirección General de Familia y Mujer.

Todas estas acciones vienen avaladas por diferentes estudios internacionales y nacionales, considerándose como viables y necesarias (Banyard et al., 2005; Grauerholz et al., 1999; Valls 2008; Valls, Flecha y Melgar, 2008), actuaciones que ya son desarrolladas en diferentes universidades inglesas y americanas.

Entre las limitaciones del estudio debemos citar el no poder abarcar a toda la población universitaria, especialmente al personal de PDI y de PAS, lo cual nos daría una visión más realista de la incidencia y cómo afecta la violencia de género en estos colectivos, caracterizados frente a los estudiantes, por ser de más edad, con familia y más años de experiencia dentro de la universidad. No podemos generalizar los resultados encontrados a toda la comunidad universitaria, así como a otras universidades. Además, puede resultar importante conocer la incidencia y la percepción que se tiene sobre la violencia de género atendiendo a los diferentes campus universitarios y áreas del conocimiento.

\section{Referencias}

1. Banyard, V., Plante, E., Ward, S., Cohn, E. S., Moorhead, C., y Walsh, W. (2005). Revisiting unwanted sexual experiences on Campus. A 12-Year Follow-up. Violence Against Women, 11, 426-446. http://dx.doi. org/10.1177/1077801204274388

2. Bolanos, C. (2003). Currículum universitario, género sensitivo e inclusivo. Revista de Ciencias Sociales, 3-4, 71-78.

3. Bosch-Fiol, E. y Ferrer-Pérez, V. A. (2012). Nuevo mapa de los mitos sobre la violencia de género en el siglo XXI. Psicothema, 24, 548-554.

4. Burgués, A., Oliver, E., Redondo, G. y Serrano, Mª (2006). Investigaciones mundiales sobre violencia de género en la universidad. XI Conferencia de Sociología de la Educación. Santander, Septiembre 2005.

5. Comisión Europea (2001). La imagen que los europeos tienen de sí mismos. Los sondeos de opinión como reflejo de la realidad. Luxemburgo: Oficina de Publicaciones Oficiales de las Comunidades Europeas.

6. Expósito, F. y Herrera, M. C. (2009). Social perception of violence against women: individual and psychosocial characteristics of victims and abusers. The European Journal of Psychology Applied to Legal Context, 1, 123-145.

7. Eyre, L. (2000). The discursive framing of sexual harassment in a University Community. Gender and Education, 12, 293-307. http://dx.doi.org/10.1080/713668301

8. Ferrer Pérez, V. A., Bosch Fiol, E., Ramis Palmer, M. C. y Navarro Guzmán, C. (2006). Las creencias y actitudes sobre la violencia contra las mujeres en la pareja: determinantes sociodemográficos, familiares y formativos. Anales de Psicología, 22, 251-259.
9. González, F. y Gimeno, A. (2009). Violencia de género: Perfil de mujeres con ayuda social. Intervención Psicosocial, 18, 165-176.

10. Grauerholz, L., Gottfried, H., Stohl, C. y Gabin, N. (1999). There's safety in number. Creating a campus advisers' network to help complainants of sexual harassment and complain receivers. Violence Against Women, 5, 960-977. http:// dx.doi.org/10.1177/10778019922181563

11. Gross, A. M., Winslett, A., Roberts, M. y Gohm, C. L. (2006). An examination of sexual violence against college women. Violence Against Women, 12, 288-300. http:// dx.doi.org/10.1177/1077801205277358

12. Heise, L., Ellsberg, M. y Gottemoeller, M. (1999). Ending violence against women. Population Reports, series L, 11. Baltimore: John Hopkins University School of Public Health.

13. Herrera, M. C., Expósito, F., Moya, M., y Houston, D. M. (2012). "Having it all": Women's Perception of Impact of Female Promotion on Threat of Domestic Violence. The Spanish Journal of Psychology, 15, 670-679. http://dx.doi. org/10.5209/rev_SJOP.2012.v15.n2.38878

14. Hirigoyen, M. F. (2006). Mujeres maltratadas. Los mecanismos de la violencia en la pareja. Barcelona: Paidós.

15. Instituto de la Mujer (2004). Denuncias por malos tratos producidos por el cónyuge o análogo. Hombres y mujeres desde el 2000 al 2004. Disponible en http://www.mtas.es/ mujer/mcifras/VIOLENCI.HTM

16. Labrador, F. J., Rincón, P., de Luís, P. y Fernández-Velasco, M. R. (2004). Mujeres víctimas de la violencia doméstica. Madrid: Pirámide.

17. Lila, M., Catalá, A., Conchell, R., García, A., Lorenzo, M. V., Pedrón, V. y Terreros, E. (2010). Una experiencia de investigación, formación e intervención con hombres penados por violencia contra la mujer en la Universidad de Valencia: Programa Contexto. Intervención Psicosocial, 19, 167-179. http://dx.doi.org/10.5093/in2010v19n2a8

18. Medina, J. (2002). Violencia contra la mujer en la pareja: Investigación comparada y situación en España. Valencia: Tirant lo Blanch.

19. Menéndez, S., Pérez, J. y Lorence, B. (2013). La violencia de pareja contra la mujer en España: Cuantificación y caracterización del problema, las víctimas, los agresores y el contexto social y profesional. Psychosocial Intervention, 2, 41-53. http://dx.doi.org/10.5093/in2013a6

20. Meras, A. (2003). Prevención de la violencia de género en adolescentes. Estudios de Juventud, 62, 143-150.

21. Montilla, G., Pérez-Cordón, L. G. y Montes-Berges, B. (2010). Towards an education aware of the gender violence problematic. Ponencia presentada en International Technology, Education and Development Conference. Valencia, Marzo 2010 (paper).

22. Montilla, G., Pérez, L. G., Castillo-Mayén, M.R. y MontesBerges, B. (2012). Buscando la educación consciente de 
la problemática de la violencia de género: Diseño de una página web. Revista Magina, 16, 123-146.

23. Muñoz-Rivas, M., Graña, J. L., O'Leary, K. D. y González, P. (2006). Agresión física y psicológica en las relaciones de noviazgo en universitarios españoles. Psicothema, 19, 102-107.

24. Nabors, E. L., Dietz, T. L. y Jasinski, J. L. (2006). Domestic violence beliefs and perceptions among college students. Violence and Victims, 21, 779-795. http://dx.doi. org/10.1891/0886-6708.21.6.779

25. Observatorio Estatal de Violencia sobre la Mujer (2011). Macroencuesta de Violencia de Género 2011. Principales resultados (8 de febrero de 2012). Disponible en http:// www.seigualdad.gob.es/violenciaGenero/documentacion/ pdf/Macroencuesta2011 Principales resultados.pdf

26. Oliver, E. y Valls, R. (2004). Violencia de género. Investigaciones sobre quiénes, por qué y cómo superarla. Barcelona: El Roure.

27. Peters, J. (2008). Measuring myths about domestic violence: Development and initial validation of the domestic violence myth acceptance scale. Journal of Aggression, Maltreatment \& Trauma, 16, 1-21. http:// dx.doi.org/10.1080/10926770801917780

28. Rentschle, C. A. (2000). Designing fear: Environmental security and violence against women. Cultural Studies: A Research, 5, 281-307.

29. Sanmartín, J., Iborra, I., García, Y. y Martínez, P. (2010). III Informe internacional violencia contra las mujeres en las relaciones de pareja. Estadísticas y legislación. Valencia: Centro Reina Sofía para el Estudio de la Violencia.
30. Smith, P. H., White, J. W. y Holland, L. (2003). A longitudinal perspective on dating violence among adolescents and collage-age women. American Journal of Public Health, 93, 104-110. http://dx.doi.org/10.2105/AJPH.93.7.1104

31. Straus, M. (2004). Prevalence of violence against dating partners by male and female university students worldwide. Violence Against Women, 10, 790-811. http://dx.doi. org/10.1177/1077801204265552

32. Unidad de Igualdad (2012). Universidad de Valencia. Disponible en http://www.uv.es/igualtat/

33. Valls, R. (2008). Violencia de género en las universidades españolas Año 2006 -2008. Madrid: Ministerio de Igualdad.

34. Valls, R., Flecha, A. y Melgar, P. (2008). Violència de gènere $\mathrm{a}$ les universitats catalanes: mesures per a la prevenció i superació. Temps d'Estudis, 35, 197-212.

35. Valls, R., Oliver, E., Sánchez, M., Ruíz, L. y Melgar, P. (2007). ¿Violencia de género también en las universidades? Investigaciones al respecto. Revista de Investigación Educativa, 25, 219-231.

36. Vázquez, E., Torres, A., Otero, P., Blanco, V. y López, M. (2010). Prevalencia y factores de riesgo de la violencia contra la mujer en estudiantes universitarias españolas. Psicothema, 22, 196-201.

Fecha de recepción: 12 de diciembre, 2103 Fecha de recepción de la versión modificada: 12 de marzo, 2014 Fecha de aceptación: 9 de junio, 2014 